\title{
High-resolution movies of molecular rotational dynamics captured with ultrafast electron diffraction
}

\author{
Yanwei Xiong ๑, Kyle J. Wilkin, and Martin Centurion $\odot$ \\ Department of Physics and Astronomy, University of Nebraska-Lincoln, Lincoln, Nebraska 68588, USA
}

(Received 14 July 2020; accepted 23 September 2020; published 12 October 2020)

\begin{abstract}
Imaging the structure of molecules during a photoinduced reaction is essential for elucidating reaction mechanisms. This requires high spatiotemporal resolution to capture nuclear motions on the femtosecond and subangstrom scale, and a sufficiently high signal level to sample their continuous evolution with high fidelity. Here we show that, using high-repetition-rate ultrafast electron diffraction, we can accurately reconstruct a movie of the coherent rotational motion of laser-aligned nitrogen molecules. We have used a tabletop 90-keV photoelectron gun to simultaneously achieve high temporal resolution of $240 \mathrm{fs}$ full width at half maximum and an electron beam current that is more than an order of magnitude above the previous state of the art in gas-phase ultrafast electron diffraction. With this, we have made an essentially continuous real-space experimental movie of the rotational motion of the molecular wave packet as it evolves from initial alignment and past multiple revivals.
\end{abstract}

DOI: 10.1103/PhysRevResearch.2.043064

\section{INTRODUCTION}

Observing the structural changes that take place during a molecular reaction remains a challenge, since it requires simultaneously reaching a high spatiotemporal resolution of sub-angstrom and femtosecond, while achieving sufficiently high signal-to-noise ratio to retrieve structural dynamics with high fidelity. Laser-based spectroscopic methods were used to probe molecular reactions on the relevant femtosecond timescales [1,2]. More recently, multiple experimental methods have been developed that are sensitive to the molecular structure, such as gas-phase ultrafast electron diffraction (UED) [3,4], ultrafast $\mathrm{x}$-ray scattering [5], Coulomb explosion imaging [6,7], and laser-induced electron diffraction [8,9]. The diffraction methods have the advantage that they are directly sensitive to the spatial distribution of charge. UED has the additional advantage that it is a weak probe; i.e., it does not disturb the system being studied. Therefore, with UED it is possible to retrieve dynamical structural information directly from the experimental data without theoretical modeling of the effect of the probe on the system. Significant effort has been devoted recently to improve the temporal resolution of UED experiments, mostly transitioning from tabletop keV setups [10-12] to relativistic mega-electron-volt $(\mathrm{MeV})$ instruments [13-16]. However, these instruments have not significantly increased the average electron beam current, which has become a limiting factor in gas-phase UED.

The first application of $\mathrm{MeV}$ electron gun technology to gas-phase UED has resulted in a temporal resolution of $230 \mathrm{fs}$ [17] and more recently 150 fs [18], compared to 850 fs for $\mathrm{keV}$ instruments [4]. This has enabled major scientific

Published by the American Physical Society under the terms of the Creative Commons Attribution 4.0 International license. Further distribution of this work must maintain attribution to the author(s) and the published article's title, journal citation, and DOI. advances such as the observation of coherent rotation and vibrational motion [17,19]; capturing the passage of a nuclear wave packet through a conical intersection [18]; retrieving transient structures and observing structural dynamics in the electronic ground state [20]; resolving a ring opening reaction [21]; and, more recently, simultaneously capturing nuclear and electronic changes [22]. The main limitation of gas-phase $\mathrm{MeV}$ UED has been the low electron beam current, which results in low signal levels and long acquisition times. In many cases low signal levels cannot be compensated by further increases in integration time due to the presence of noise that increases with time, such as detector background. This has become a significant challenge due to the limited beam time that is generally available at user facilities and has so far prevented systematic studies, such as exploring the wavelength dependence of the reaction dynamics or carrying out a set of experiments on similar molecules. These systematic measurements are essential to elucidate the general rules underlying photochemical reactivity.

\section{EXPERIMENT}

Here, we demonstrate a tabletop kilo-electron-volt $(\mathrm{keV})$ gas-phase UED setup with a temporal resolution of $240 \mathrm{fs}$ full width at half maximum (FWHM) and an electron beam current that is more than an order of magnitude higher than that achieved at MeV-UED facilities. The high beam current will enable studies beyond those currently possible, and the small scale of the instrument makes it suitable for a university laboratory and can thus increase accessibility. The higher beam current is reached by running at a high repetition rate of $5 \mathrm{kHz}$, which is made possible by the use of a dc accelerator. In order to reach femtosecond resolution, a dc-rf electron gun is employed where electrons are first accelerated in a dc electric field and then temporally compressed at the sample using an rf cavity [23-25]. Using a dc accelerator has the advantage that the gun can be run at high repetition rates, well beyond $5 \mathrm{kHz}$, limited only by the repetition rate of the 


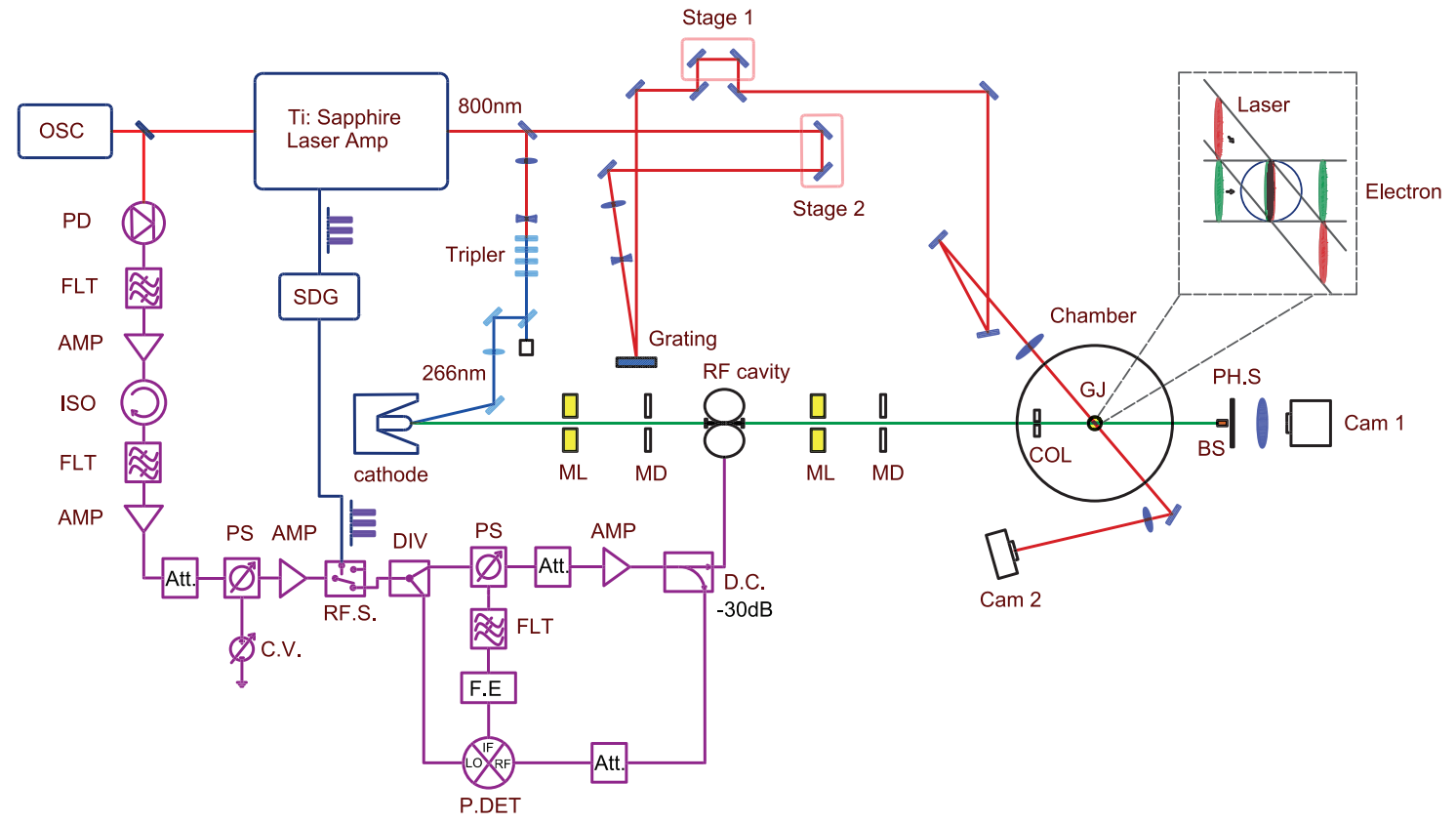

FIG. 1. Diagram of experimental layout, not to scale. The path drawn in red corresponds to the 800-nm-wavelength laser pulse. A fraction of the laser is converted to the third harmonic at $267 \mathrm{~nm}$ to drive the photocathode, shown here in light blue. The path drawn in green corresponds to the electron beam trajectory from the photocathode to the detector. Camera 1 captures the light generated by the electrons impinging on the phosphor screen. Camera 2 is used to monitor the laser pulse. The path in purple corresponds to the 3-GHz rf signal used to drive the rf cavity. The dark blue path is the electric $5-\mathrm{kHz}$ trigger signal from the laser control unit to control the release timing of the laser pulse and the 3-GHz pulse. The inset shows a sketch of how the velocity mismatch is compensated at the interaction region using a laser pulse with a tilted intensity front traveling at an angle with respect to the electron beam. OSC $=$ oscillator, PD $=$ photodiode, $\mathrm{FLT}=$ filter, $\mathrm{AMP}=$ amplifier, $\mathrm{ISO}=$ isolator, $\mathrm{SDG}=$ synchronization and delay generator, Att. $=$ attenuator, $\mathrm{RF} . \mathrm{S}=\mathrm{rf}$ switch, $\mathrm{DIV}=$ power divider, $\mathrm{PS}=$ phase shifter, C.V. = control voltage, F.E. = feedback electronics, P.DET $=$ phase detector, D.C. $=$ directional coupler, $\mathrm{ML}=$ magnetic lens, $\mathrm{MD}=$ magnetic deflector, $\mathrm{COL}=$ collimator, $\mathrm{GJ}=$ gas jet, $\mathrm{BS}=$ beam stop, $\mathrm{PH} . \mathrm{S}=$ phosphorus screen, and $\mathrm{Cam}=$ camera.

laser and the frequency of the rf cavity ( $3 \mathrm{GHz}$ in our case). While dc-rf setups have been used for diffraction experiments in solids, gas-phase experiments present additional challenges due to the low scattering intensity and extended size of the target. In particular, the velocity mismatch between laser and electron pulses causes their relative time delay to vary as they traverse the sample, resulting in a degradation of the temporal resolution. We have used a laser pulse with a tilted wavefront to excite the sample in a geometry that compensates for the velocity mismatch between laser and electrons [26,27]. We have applied this setup to capture the rotational dynamics of impulsively aligned nitrogen $\left(\mathrm{N}_{2}\right)$ molecules. While previous UED experiments have demonstrated that UED can capture the angular distribution of aligned $\mathrm{N}_{2}$ molecules [17], the measurement was limited to a few frames of the motion due to the low electron beam current. The high beam current has allowed us to record a large number of frames with high signal levels, essentially following the dynamics continuously. The performance demonstrated here will be transformative for gas UED because it will enable experiments with more complex molecules and systematic studies of structural dynamics that are currently not possible due to low signal levels.

The rotational dynamics of laser-aligned small molecules has been investigated widely using a variety of probing mechanisms [28-32], partly as a testbed of experimental methods because the rotational motion can be simulated accurately and compared with experiments. UED is a direct structural probe sensitive to the spatial distribution of the nuclei, thus allowing the full angular distribution to be extracted from the measurement. Furthermore, when probing structural dynamics in photoexcited molecules, UED presents the advantage that it is a weak probe; i.e., it does not expose the sample to strong fields.

The instrument layout, shown in Fig. 1, is described in detail in Appendixes A and B. Briefly, the setup comprises four major parts: the laser system, the electron beamline, the interaction region, and the synchronization mechanism. The laser pulses $(50 \mathrm{fs}, 800 \mathrm{~nm}, 2 \mathrm{~mJ})$ are produced at a repetition rate of $5 \mathrm{kHz}$ and are used to impulsively align the target molecules and to drive the photoelectron gun after frequency tripling to a wavelength of $267 \mathrm{~nm}$. The electron pulses are generated in a photocathode and quickly accelerated to 90 $\mathrm{keV}$ in a dc electric field, guided by electron optics and temporally compressed on the sample using an rf cavity. The synchronization of the rf fields and the laser is achieved with a homemade synchronizer based on the design described in Otto et al. [25]. This synchronizer allows us to operate the experiment for several hours with high stability. The temporal resolution can be significantly deteriorated by the velocity mismatch between laser and electron pulses, since it induces a change in the relative time delay between the pulses across the sample volume. We have mitigated this issue by using a laser pulse with a tilted wavefront and an angled incidence to 
the electron beam such that the longitudinal component of the laser velocity is matched to the speed of the electrons [27]. The laser pulse with a tilted pulse front has a time duration of $60 \mathrm{fs}$ and energy of $1 \mathrm{~mJ}$, and is focused to a spot size of $190 \mu \mathrm{m}$ (horizontal) $\times 260 \mu \mathrm{m}$ (vertical) on the sample. The electron beam delivers on the sample 10000 electrons per pulse ( $8 \mathrm{pA}$ of average current) with a $100-\mu \mathrm{m}$ diameter. A higher electron beam current is possible with a larger beam, but in this case the electron beam size was reduced with an aperture to probe a region with approximately uniform laser intensity. Nitrogen molecules are delivered to the interaction as a supersonic molecular beam, $200 \mu \mathrm{m}$ in diameter, generated by a de Laval nozzle with an inner diameter of $30 \mu \mathrm{m}$ supplied with a backing pressure of 1200 mbar of nitrogen. The scattered electrons are captured using a phosphor screen that is imaged onto an electron-multiplying charge-coupled device (EMCCD) camera. Our instrument combines pulse compression and velocity mismatch compensation, which allows us to reach an instrument response time of $240 \mathrm{fs}$, with a low timing drift of $50 \mathrm{fs}$ rms over several hours.

\section{THEORY}

The laser induced impulsive alignment of linear molecules (more detail in Appendix C) can be described by the timedependent Schrödinger equation and numerically solved using spherical harmonics $[33,34]$. The calculated angular distribution is used to construct a simulated diffraction pattern at each time point. Although our measurement provides the full angular distribution, for simplicity we use the expectation value of $\left\langle\cos ^{2} \alpha\right\rangle$ to describe the degree of alignment, where $\alpha$ is the angle between the laser polarization (the alignment axis) and the axis of the molecule.

The diffraction intensity for a sample of molecules in the gas phase can be calculated using an incoherent sum of the scattering pattern of each molecule. A general description of the scattering can be found in Appendix C. For the special case of an ensemble of laser-aligned nitrogen molecules with an angular distribution $g(\alpha)$, the total diffraction intensity captured on a flat detector can be written as

$$
\begin{aligned}
I_{\text {Total }}\left(s_{x}, s_{y}\right)= & 2\left|f_{N}(s)\right|^{2}+2 \iint\left|f_{N}(s)\right|^{2} \cos \left[\vec{s} \cdot \vec{r}\left(\alpha_{0}, \beta_{0}\right)\right] \\
& \times g\left(\alpha_{0}\right) \sin \alpha_{0} d \alpha_{0} d \beta_{0}=I_{\mathrm{at}}+I_{\mathrm{mol}}
\end{aligned}
$$

where $f_{N}$ is the complex scattering amplitude of the nitrogen atom; $\vec{s}$ is the momentum transfer vector with magnitude $s=\frac{4 \pi}{\lambda} \sin \left(\frac{\theta}{2}\right) ; \vec{r}$ is the vector pointing in the direction of the molecular bond; $\alpha_{0}, \beta_{0}$ are the polar and azimuthal angles with respect to the alignment axis. The first term in the sum, the atomic scattering intensity $I_{\text {at }}$, does not contain structural or angular information. The molecular scattering intensity $I_{\text {mol }}$ depends on both the structure and angular distribution of the molecules. In the experiment, we use the background-subtracted (difference) scattering signal to remove the contribution from the atomic scattering and other background sources:

$$
\begin{aligned}
\Delta I_{\mathrm{mol}}\left(s_{x}, s_{y}, t\right) & =I_{\mathrm{Total}}\left(s_{x}, s_{y}, t\right)-I_{\mathrm{Total}}^{\mathrm{Random}}\left(s_{x}, s_{y}\right) \\
& =I_{\mathrm{mol}}\left(s_{x}, s_{y}, t\right)-I_{\mathrm{mol}}^{\mathrm{Random}}\left(s_{x}, s_{y}\right),
\end{aligned}
$$

where $I_{\text {Total }}^{\text {Random }}$ is a diffraction pattern from randomly oriented molecules, which was measured by setting the time delay such that the electrons arrive at the sample before the laser. By still having the laser on we capture any background noise produced by scattered laser light on the detector. $I_{\text {mol }}\left(s_{x}, s_{y}, t\right)$ can then be reconstructed by adding the contribution of $I_{\mathrm{mol}}^{\text {Random }}$, which is calculated from the known nitrogen bond distance. In diffraction from randomly oriented molecules, the pair distribution function (PDF), which has a peak corresponding to each interatomic distance in the molecule, is typically used to analyze the diffraction data. The two-dimensional (2D) inverse Fourier transform of the measured diffraction pattern followed by an Abel inversion gives a modified pair distribution function (MPDF), which is a scaled and angle-resolved version of the PDF, and represents the three-dimensional probability density of the nuclear wave packet:

$$
\begin{aligned}
\operatorname{MPDF}(r, \alpha, t) & =\operatorname{Abel}^{-1} \mathrm{FT}_{2 D}^{-1}\left[I_{\mathrm{mol}}\left(s_{x}, s_{y}\right)\right] \\
& =g(\alpha, t) \operatorname{PDF}(r) \otimes \frac{\mathrm{FT}^{-1}\left[\left|f_{N}(s)\right|^{2}\right]}{r_{N}},
\end{aligned}
$$

where $r_{N}$ is the bond distance, FT is the Fourier transform, and $\otimes$ denotes a convolution and $\operatorname{PDF}(r)=\frac{\delta\left(r-r_{N}\right)}{r_{N}} \otimes H(r)$, in which $H(r)$ is a function that depends on the maximum value of $s$ in the measurement and determines the width of the peaks in the PDF. The MPDF contains information on both the bond length and the angular distribution, and can be thought of as an image of the molecular ensemble at time $t$.

\section{RESULTS}

A femtosecond laser pulse is used to excite a rotational wave packet. The maximum alignment is reached a few hundred femtoseconds after the impulsive laser excitation, followed by a dephasing and subsequent revivals of the alignment. The main signature of alignment in the diffraction pattern is the appearance of anisotropy, which contrasts against the circularly symmetric diffraction pattern of randomly oriented molecules. First, we have used the time evolution of the anisotropy to characterize the temporal resolution and timing drift of the experimental setup. The fast-changing angular distribution provides a good metric to determine the instrument response time (temporal resolution) by comparing theory and experiment, given that the rotational dynamics of diatomics can be simulated very accurately. The instrument response time determined in this way reflects the true temporal resolution of the experiment as it includes contributions from both the laser and electron pulse durations, the effect of velocity mismatch, and time of arrival jitter between laser and electron pulses.

Figure 2 shows the temporal evolution of the anisotropy in the diffraction patterns. The anisotropy is defined as $\left(S_{H}-S_{V}\right) /\left(S_{H}+S_{V}\right)$ where $S_{H}$ and $S_{V}$ are the sum of the counts in horizontal and vertical cones in $I_{\text {mol }}$, respectively, ranging from 3 to $4.5 \AA^{-1}$. The time $t=0$ was set to overlap with the prompt alignment peak. At each time step, a diffraction pattern was recorded with an acquisition time of $25 \mathrm{~s}$. The instrument response time was determined by fitting a theoretical alignment scattering signal to the data using four parameters: the laser fluence, the initial rotational temperature 


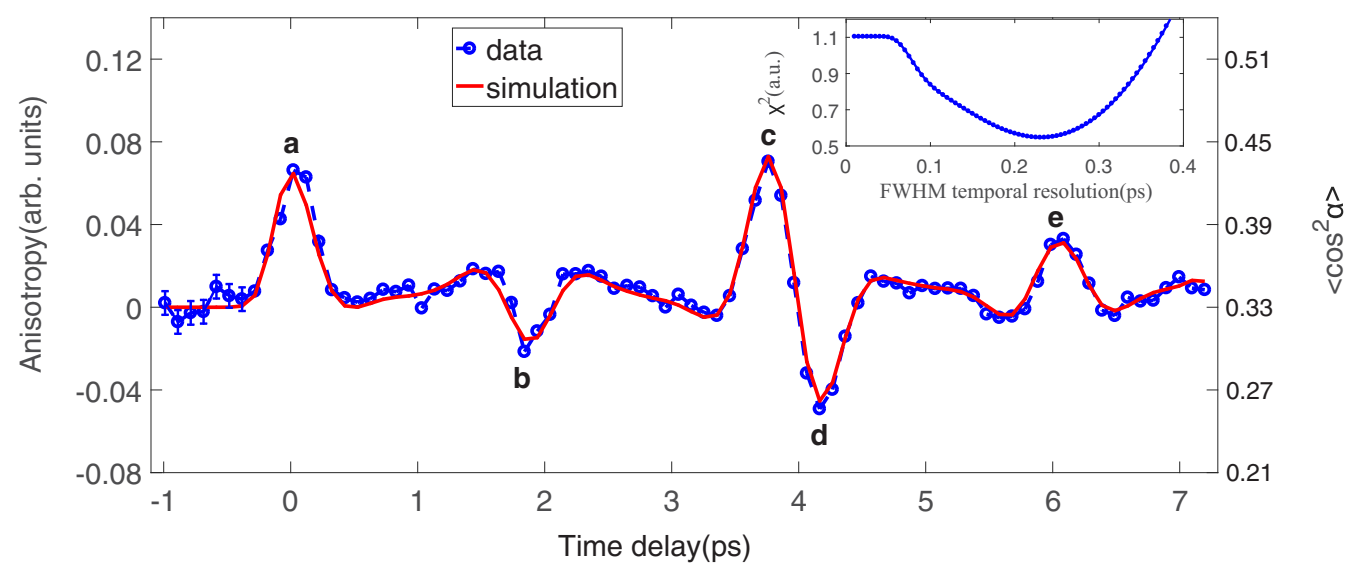

FIG. 2. Temporal evolution of the $\mathrm{N}_{2}$ rotational wave packet. The anisotropy experimental and simulated diffraction patterns are shown in blue and red, respectively. The exposure time for each data point is $25 \mathrm{~s}$. The inset shows the $\chi^{2}$ value of the fit as a function of the FWHM temporal resolution. Statistical error bars for the first seven points, which is the standard deviation of anisotropy of ten images before the first peak, are used to show the uncertainty of measurement at each data point. The right axis shows the $\left\langle\cos ^{2} \alpha\right\rangle$ values, as defined in the text.

of the molecules, the instrument response time, and a rescaling factor that accounts for the spatial overlap of the beams and the variation of the laser intensity across the sample. The instrument response term is simulated by convolving the theoretical signal with a Gaussian function with a FWHM that reflects the temporal resolution of the experiment. In order to compare the theory and experiment, simulated diffraction patterns are generated using the simulated angular distributions, and then the anisotropy is calculated in the same manner as in the experiment. The best fit to the data returned a laser fluence of $1.7 \mathrm{~J} / \mathrm{cm}^{2}$, rotational temperature of $45 \mathrm{~K}$, rescaling factor 0.35 , and instrument response time of $240 \mathrm{fs}$. The measured laser fluence is $1.78 \mathrm{~J} / \mathrm{cm}^{2}$ and the rotational temperature was estimated to be $43 \mathrm{~K}$ using the supersonic expansion model [35], both in good agreement with the fit values. The left axis shows the anisotropy in arbitrary units, and the right axis shows the value of $\left\langle\cos ^{2} \alpha\right\rangle$. The maximum degree of alignment was measured at $\left\langle\cos ^{2} \alpha\right\rangle=0.44$ and variations on the order of 0.01 are well resolved, which serves to highlight the high sensitivity of UED. The experiment was repeated four times and showed consistent results in the retrieved temporal resolutions of 240, 240, 230, and 250 fs. A key factor in maintaining an optimized temporal resolution was to keep the electron beam current (which is measured in our experiment by the beam stop) constant to within $10 \%$ of the set value. Larger fluctuations led to degradation of the temporal resolution. We have also characterized the stability of the timing by measuring changes in the position of the alignment peaks over several hours. The measured timing drift over $4.5 \mathrm{~h}$ was $50 \mathrm{fs}$ rms, which is comparable to state of the art facilities such as the MeV-UED setup at SLAC [36].

We now focus on retrieving the time-dependent angular distribution and imaging of the rotational wave packet. Figures 3(a)-3(e) show the 2D diffraction-difference signals $\Delta I_{\text {mol }}\left(s_{x}, s_{y}, t\right)$ at five different times: the prompt alignment peak, the first quarter revival, the peak and trough corresponding to the half-revival, and the peak at the $\frac{3}{4}$ revival. Note that at the half revival, the distribution changes completely from prolate to oblate in around $300 \mathrm{fs}$, and the change is very well resolved by the measurement. Difference-diffraction patterns in Fig. 3 were generated by averaging four different scans at a total integration time of $100 \mathrm{~s}$ per time step. In contrast, a similar experiment carried out by Yang et al. at the SLAC MeV-UED setup [17,37] using an electron beam current of $0.7 \mathrm{pA}(37500 \mathrm{e} /$ pulse at a repetition rate of $120 \mathrm{~Hz})$ required integration times between 60 and 90 min to achieve comparable high-quality diffraction-difference images. This large reduction in acquisition times is accomplished by using a higher beam current and better matching of electron and laser spot size, which we achieved using an aperture to reduce the electron beam size on the sample. Moreover, the increased signal-to-noise ratio (SNR) and fine time sampling of our experiment allows us to retrieve a full movie of the dynamics, essentially following the nuclear motion continuously and retrieving the full angular distribution at each step.

Figures 3(f)-3(j) show the corresponding MPDFs. The data analysis procedure is described in detail in Appendix D. Briefly, the diffraction patterns are normalized, filtered to remove noise, and the four quadrants are averaged, taking advantage of the symmetry. The area at the center where the scattering is blocked by the beam stop is filled in by linear interpolation to zero at $s=0$. The MPDF is retrieved by first adding the contribution of randomly oriented molecules back into the $\Delta I_{\mathrm{mol}}$, which isolates the contribution of excited molecules. The resulting pattern then goes through a 2D inverse Fourier transform followed by Abel inversion. The experimental MPDFs shown in Figs. 3(f)-3(j) display the spatially resolved motion of the coherent rotational wave packet, which is in good agreement with the theoretical MPDFs shown in Figs. 3(k)-3(o). The angular distribution can be extracted by analyzing the MPDF at a fixed radius corresponding to the interatomic distance. We can see in Fig. 3(f) that the rotational wave packet is localized along the direction of laser excitation, as expected for the peak of the alignment. The angular distribution reflects the degree of alignment, which is relatively broad. In addition to the MPDFs at other key times shown in Fig. 3, we have retrieved a movie of the MPDF which covers the initial alignment through the revivals up to about 7 ps (see Supplemental Material, Movie M1 [38]). The movie shows the continuous three-dimensional motion of the 

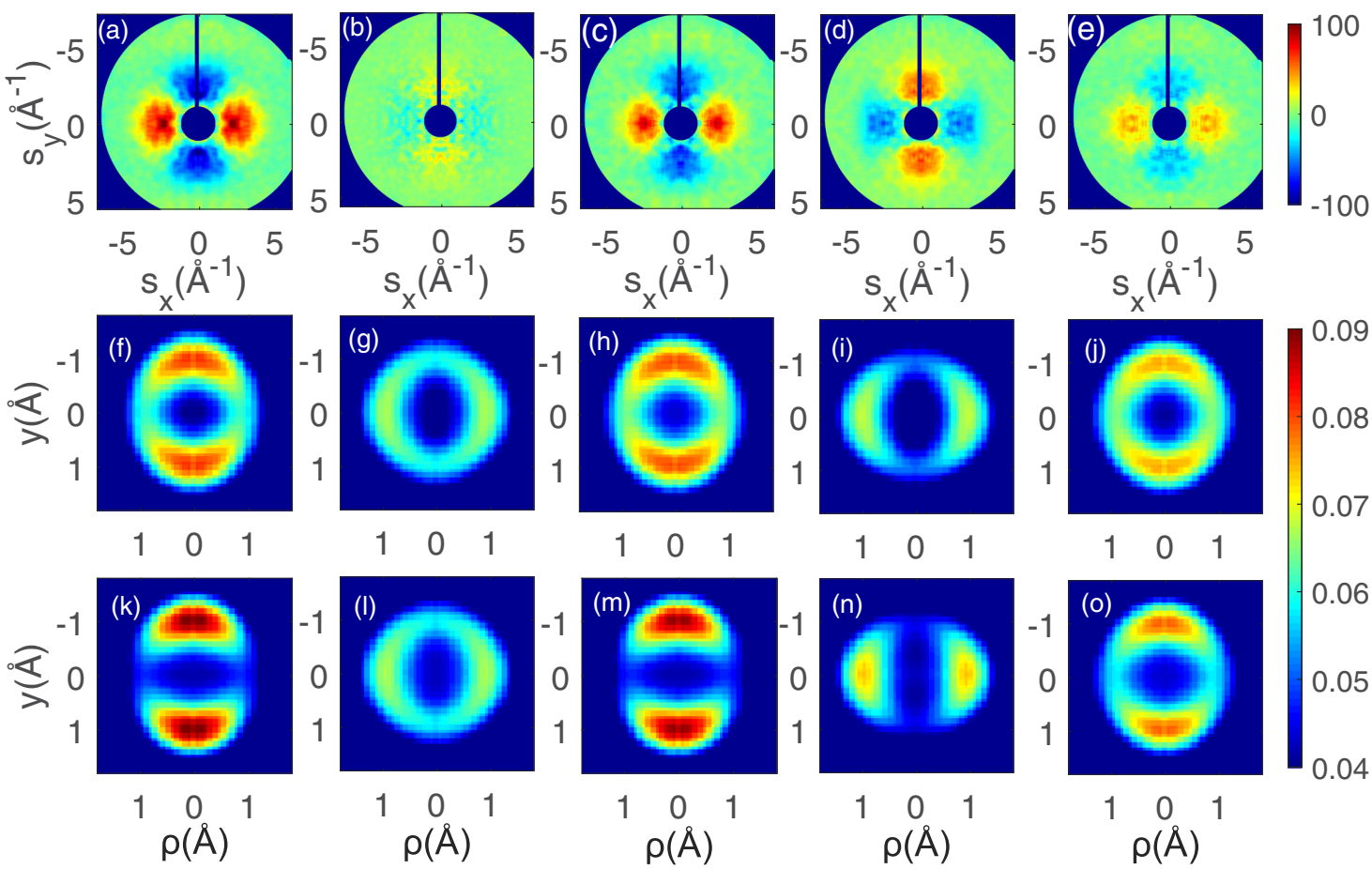

FIG. 3. $\Delta I_{\mathrm{mol}}\left(s_{x}, s_{y}, t\right)$ Difference-diffraction pattern corresponding to the features marked in Fig. 2, at delay times (a) $t=0 \mathrm{ps,} \mathrm{(b)} t=1.9$ ps, (c) $t=3.8 \mathrm{ps,} \mathrm{(d)} t=4.2 \mathrm{ps,} \mathrm{and} \mathrm{(e)} t=6.1 \mathrm{ps}$. The dark circle and vertical line correspond to the regions where scattered electrons are blocked by the beam stop. (f-j) The experimental modified pair distribution function MPDF retrieved from the above pattern in parts (a-e). $(\mathrm{k}-\mathrm{o})$ The simulated MPDF calculated for the same time points as the data.

nuclear wave packet as retrieved from the data, which is in good agreement with the simulation (shown in the Supplemental Material as Movie M2 [38]).

Figure 4 shows the reconstructed angular distribution at the first alignment peak, along with the simulated curve, and the simulated curve convolved with the instrument response time. The angular distribution is calculated from the transform of the diffraction pattern, with a baseline added to normalize the distribution. There is good agreement between the experimental and simulated angular distributions, with improved agreement after accounting for the temporal resolution. The full rotational dynamics are clearly displayed

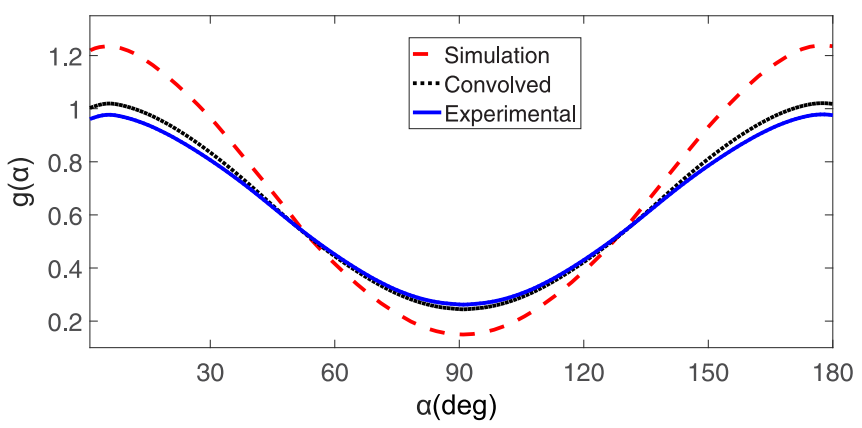

FIG. 4. Experimental (blue) and simulated (red) angular distributions $g(\alpha)$ calculated from Fig. 3 for the diffraction pattern at $t=0$ ps. To account for the instrument response, the simulated angular distribution was convolved with a Gaussian function with FWHM of $240 \mathrm{fs}$ (black). in Fig. 5(a), where the angular distribution $g(\alpha, t)$ is shown as a function of $\alpha$ (horizontal axis) and time $t$ (vertical axis). This representation clearly displays the fast changes in the angular distribution. Figure 5(b) shows the theoretical angular distribution extracted from the alignment simulations, convolved with the temporal resolution of the experiment. The experiment accurately captures all the main features of the dynamics, including small changes in the distribution, and is in very good agreement with the theory.

\section{CONCLUSION}

In summary, we report on a continuous experimental movie of the impulsive alignment of nitrogen, captured using a highrepetition rate dc-rf keV UED instrument. This measurement demonstrates the ability of a tabletop keV UED to achieve spatiotemporal resolutions comparable to those reported by MeV-UED experiments in the gas phase. Our setup provides higher signal levels and much shorter acquisition times thanks to an order of magnitude increase in average electron beam current. We believe this increase in data rate and quality to be transformative to gas-phase UED, as it enables better sampling of the dynamics; the investigation of additional reaction parameters, such as excitation conditions (laser wavelength, pulse energy); and comprehensive studies on how the photochemistry of a class of molecules is affected by the addition of structural motifs and functional groups. These systematic studies will be crucial to gain insights into the underlying rules governing reaction dynamics. 
(a)

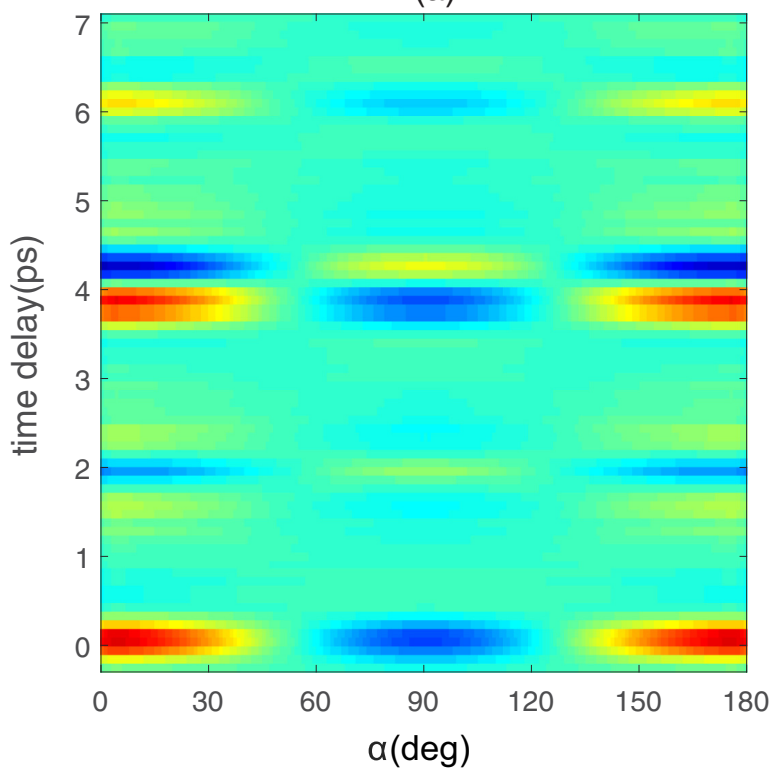

(b)

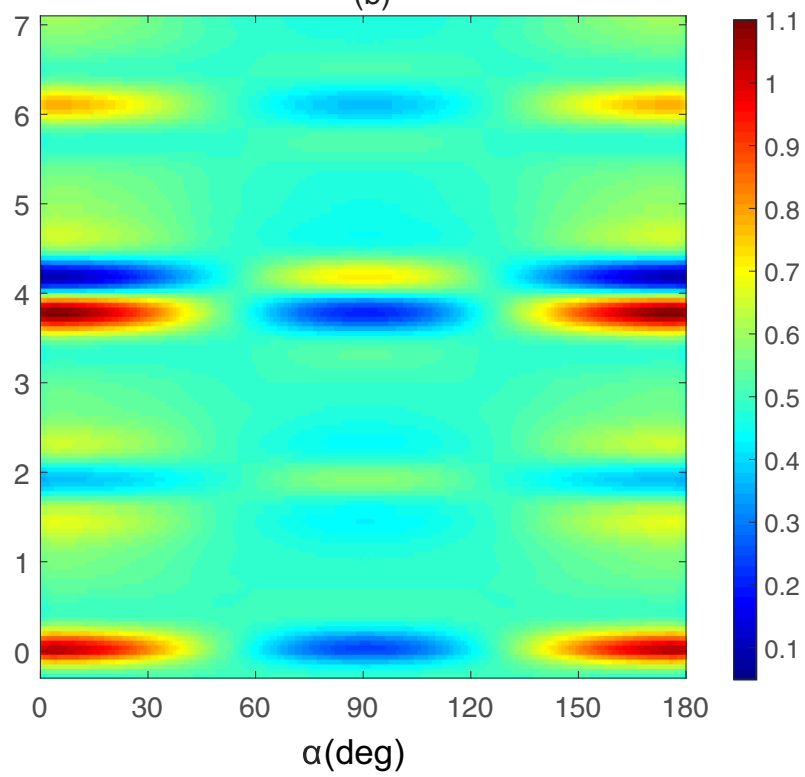

FIG. 5. (a) Time evolution of angular distribution $g(\alpha, t)$ calculated from experiment data. (b) Simulation of $g(\alpha, t)$ convolved with a 240 -fs FWHM instrument response function.

\section{ACKNOWLEDGMENTS}

This work was supported by the National Science Foundation, Physics Division, Atomic, Molecular and Optical Sciences program, under Award No. PHY-1606619.

\section{APPENDIX A: EXPERIMENTAL SETUP}

Figure 1 shows a layout of the experimental setup, which comprises four major parts: the laser system, the electron beamline, the interaction region, and the synchronization mechanism. The laser system consists of a mode-locked Ti:sapphire oscillator running at a repetition rate of $75 \mathrm{MHz}$ followed by an amplifier that produces pulses with a duration of $50 \mathrm{fs}$, central wavelength of $800 \mathrm{~nm}$, and pulse energy of $2 \mathrm{~mJ}$ at a repetition rate of $5 \mathrm{kHz}$. The laser pulse is split into two parts, with most of the energy going to pump the molecules, in this case to trigger the impulsive alignment. A small fraction of the laser pulse energy is frequency tripled and used to trigger the photocathode, which synchronizes the photoemission to the laser. The electron beamline consists of photocathode, a dc electric field that accelerates the electrons to a kinetic energy of $90 \mathrm{keV}$ (electron speed is $0.526 c$, where $c$ is the speed of light), magnetic focusing and steering electron optics, and an rf cavity that modifies the velocity distribution of the electrons such that the pulse will longitudinally compress after the cavity, reaching a minimum pulse duration at the target position. The distance from the photocathode to the rf cavity is $580 \mathrm{~mm}$ and the distance from the rf cavity to the molecular target is $220 \mathrm{~mm}$. In the interaction region, molecules are introduced in a supersonic beam using a de Laval nozzle with an inner diameter of $30 \mu \mathrm{m}$ and a backing pressure of 1200 mbar of nitrogen. The electron beam intersects the gas jet at a distance of approximately $200 \mu \mathrm{m}$ from the tip of the nozzle. The diameter of the gas jet at the interaction region is approximately $200 \mu \mathrm{m}$. An aperture (14 $\mathrm{mm}$ upstream of the sample) was used to reduce the electron beam size to $100 \mu \mathrm{m}$ FWHM. The number of electrons on the sample is 10000 per pulse, or about $8 \mathrm{pA}$ of current. Since the aperture is placed close to the sample, we do not expect that is has a significant effect on the electron pulse duration, and thus a higher charge could be delivered on the sample if a larger beam is used, up to about 100000 electrons per pulse or a current of $80 \mathrm{pA}$.

The scattered electrons are recorded using a phosphor screen optically coupled to an EMCCD. The directly transmitted (unscattered) electron beam is blocked before the screen to prevent saturation of the images.

The velocity mismatch between the pump laser and the probe electron beam, traveling at speeds significantly below the speed of light, can result in a degradation of temporal resolution. As the electron pulse lags behind the pump pulse, molecules closer to the electron source experience a shorter pump-probe delay than those farther away, thus resulting in a temporal smearing of the measurement. To prevent this temporal blurring, we deliver the laser pulse at $58^{\circ}$ with respect to the electron beam direction, and we also tilt the intensity front of the laser pulse by this same angle. In this manner the intensity front and the longitudinal speed of both pulses are matched and all molecules in the interaction region experience the same time delay between the laser and electron pulses. The tilt angle and the duration of the tilted laser pulse are measured in situ using a reference laser pulse [27], omitted from Fig. 1 for simplicity. The spatial and temporal overlap of the laser and electron pulses is determined with the aid of the plasma lensing effect [37]. The laser pulse with a tilted pulse front has a time duration of $60 \mathrm{fs}$, energy of $1 \mathrm{~mJ}$, and is focused to a spot size of 190 um (horizontal) $\times 260$ um (vertical) on the sample.

To maintain the synchronization between pump and probe, and prevent drifts in the time of arrival of the electron beam 
at the interaction region, the time-dependent electric field of the rf cavity is synchronized with the laser. We have used a homemade synchronization system inspired by the system described in Otto et al. [25]. A small fraction of the power from the laser oscillator is filtered to select the $3-\mathrm{GHz}$ harmonic and then amplified in multiple stages to drive the rf cavity. A phase shifter is used to control the phase of the signal going into the rf cavity. The signal is split and a feedback loop is used to correct phase shifts introduced during amplification. This stabilization system, combined with environmental controls in the lab, has allowed us to reach timing drifts of $50 \mathrm{fs}$ rms over several hours, which is comparable to those achieved at state-of-the-art facilities.

\section{APPENDIX B: RF COMPRESSION AND SYNCHRONIZATION}

The electron pulses are compressed by the longitudinal electric field of the rf cavity. The cavity electric field reverses the linear longitudinal phase-space distribution of the electron bunch by decelerating the electron pulse when it enters the cavity and accelerating when it exits. The homemade synchronization system is inspired from the method in [25]. The diagram of the $\mathrm{rf}$ compression system is shown in Fig. 1. A small fraction of the power from the laser oscillator (8 $\mathrm{mW}$ ), running with a repetition rate of $75 \mathrm{MHz}$, is focused onto a fast photodiode (Newport 818-BB45). The signal from the photodiode is run through a rough bandpass filter (MiniCircuits VBF-2900+), followed by a cavity bandpass filter with a bandwidth of $80 \mathrm{MHz}$ (Anatech AB3000B509), which is used to select the 3-GHz signal with a narrow band. Three low phase noise amplifiers (one Holzworth HX2400, two HMC8411LP2FE) are used to amplify the signal to $16 \mathrm{dBm}$. A phase shifter (Analog Devices HMC928LP5E), controlled by an analog voltage, is used to adjust the phase of the electric field in the rf cavity relative to the arrival of the electron pulse. An rf switch (ZASW-2-50DRA+), triggered by a signal from the Coherent synchronization and delay generator (SDG), is used to chop the continuous if signal to a pulsed signal with a duty cycle of $5 \%$. The pulsed rf power is split into two paths, making a loop to correct the phase change introduced by the high-power amplifier (Microwave Amps AM83-3S-50-53R). The first path (high power) is sent into the rf cavity through a directional coupler (e-MECA 722N-30-3.100), and a small part of the power is extracted and used as a reference with a phase detector (Holzworth HX3400). The phase detector output voltage is used as the feedback to a phase shifter in the first path to fix the phase. We use a commercial rf cavity (AccTec BV) whose temperature is stabilized within $1 \mathrm{mK}$ rms with a temperature controller. Phase stable cables (LL142 Harbour) are used to deliver the $3-\mathrm{GHz}$ signals. The feedback electronics amplifies the phase detector output voltage with an offset voltage and feeds the voltage back to the phase shifter to compensate phase change. The function is expressed as $V_{\text {shift }}=A\left(V_{\text {detector }}+V_{\text {offset }}\right)$. The gain factor $A=-1 / k_{1} k_{2}$, where $k_{1}$ is the slope of phase to control voltage of the phase shifter, $k_{2}$ is the slope of the output voltage to phase change of the phase detector. An operational amplifier (LT1028) is used in the feedback electronics, which provides a minimum response time of $1 \mu \mathrm{s}$.

\section{APPENDIX C: THEORY OF ELECTRON DIFFRACTION FROM ALIGNED MOLECULES}

The laser induced impulsive alignment of linear molecules can be described by the time-dependent Schrödinger equation with the effective Hamiltonian $[33,34]$.

$$
\begin{aligned}
i \hbar \frac{\partial \Psi(t)}{\partial t} & =H(t) \Psi(t), \\
H(t) & =\hbar^{2} B_{e} J^{2}-\frac{1}{4} \mathcal{E}^{2}(t) \Delta \mathrm{P}^{2} \cos ^{2} \theta .
\end{aligned}
$$

The equations can be numerically solved using an expansion in the spherical harmonics $\Psi_{J_{i} M_{i}}(t)=\sum_{J} c_{J}(t)|J, M\rangle$, where $\left|J_{i} M_{i}\right\rangle$ are initial states in a Boltzmann distribution $W_{J_{i} M_{i}}(T)$ determined by the initial temperature, where $T$ is the temperature, $B_{e}$ is the rotational constant, $P$ is the polarizability, and $\mathcal{E}$ is the laser electric field. The coefficients are used to calculate the angular distribution of nitrogen molecules, and this distribution is used to construct a simulated diffraction pattern at each time point. Although our measurement provides the full angular distribution, for simplicity we use the expectation value of $\cos ^{2} \alpha$ to describe the degree of alignment, where $\alpha$ is the angle between the laser polarization (the alignment axis) and the axis of the molecule.

$$
\left\langle\cos ^{2} \alpha\right\rangle(t)=\sum_{i} \mathrm{~W}_{J_{i} M_{i}}(T)\left\langle\Psi_{J_{i} M_{i}}(t)\left|\cos ^{2} \alpha\right| \Psi_{J_{i} M_{i}}(t)\right\rangle .
$$

Here we show how the angular distribution of the molecules can be extracted from the measured twodimensional diffraction intensity. For a general function $u(\vec{s})$, the Abel transform of $\mathrm{FT}_{3 D}^{-1}[u(\vec{s})]$ is

$$
\begin{aligned}
\int_{-\infty}^{+\infty} d z \mathrm{FT}_{3 D}^{-1}[u(\vec{s})]= & \int_{-\infty}^{+\infty} d z \iiint d s_{x} d s_{y} d s_{z} u(\vec{s}) \\
& \times e^{i\left(s_{x} x+s_{y} y+s_{z} z\right)} \\
= & \iiint d s_{x} d s_{y} d s_{z} u(\vec{s}) e^{i\left(s_{x} x+s_{y} y\right)} \delta\left(s_{z}\right) \\
= & \iint d s_{x} d s_{y} u\left(s_{x}, s_{y}, s_{z}=0\right) e^{i\left(s_{x} x+s_{y} y\right)},
\end{aligned}
$$

and (1a) can be written in the inversion form when $\mathrm{FT}_{3 D}^{-1}[u(\vec{s})]$ is symmetric to an axis normal to $z$,

$$
\mathrm{FT}_{3 D}^{-1}\left[u\left(s_{x}, s_{y}, s_{z}\right)\right]=\mathrm{Abel}^{-1} \mathrm{FT}_{2 D}^{-1}\left[u\left(s_{x}, s_{y}, s_{z}=0\right)\right] .
$$

The diffraction intensity is an incoherent sum of scattering from individual molecules, in which electron waves scattered from atoms interfere. The total elastic scattering intensity from a single molecule can be written as

$$
I^{\vec{a}}(\vec{s})=\sum_{j=1}^{n} \sum_{k=1}^{n} f_{j}^{*}(s) f_{k}(s) e^{-i \vec{s} \cdot \vec{r}_{j k}(\vec{a})},
$$

where $f_{k}(s)$ is the complex scattering amplitude of the $k$ th atom, $n$ is the total number of atoms in the molecule, $\vec{s}$ is the momentum transfer vector with magnitude $s=\frac{4 \pi}{\lambda} \sin \left(\frac{\theta}{2}\right), \vec{r}_{j k}$ is the vector pointing from the $j$ th atom to the $k$ th atom, and $\vec{a}$ is the vector that indicates the orientation of the vector $\vec{r}_{j k}$. 
The terms in Eq. (C4a) where $j=k$ correspond to the atomic scattering intensity, while the $j \neq k$ terms, called molecular scattering intensity, represent the interference of atomic pairs. The diffraction intensity of an ensemble of molecules is a summation over molecules with all orientations. Here we focus on the molecular scattering. For one-dimensional alignment of molecules by a linear polarized laser with the angular distribution $g(\alpha)$,

$$
I_{\mathrm{mol}}(\vec{s})=\iint \sum_{j=1}^{n} \sum_{k=1, j \neq k}^{n} f_{j}^{*}(s) f_{k}(s) e^{-i \vec{s} \cdot \vec{r}_{j k}\left(\alpha_{0}, \beta_{0}\right)} g_{j k}\left(\alpha_{0}\right) \sin \alpha_{0} d \alpha_{0} d \beta_{0} .
$$

The inverse Fourier transform of $I_{\mathrm{mol}}(\vec{s})$ is

$$
\mathrm{FT}_{3 D}^{-1}\left[I_{\mathrm{mol}}(\vec{s})\right]=\sum_{j=1}^{n} \sum_{k=1, j \neq k}^{n} \iint g_{j k}\left(\alpha_{0}\right) \sin \alpha_{0} \delta\left[\vec{r}-\vec{r}_{j k}\left(\alpha_{0}, \beta_{0}\right)\right] d \alpha_{0} d \beta_{0} \otimes F_{j}(-\vec{r}) \otimes F_{k}(\vec{r}),
$$

where $\alpha_{0}$ and $\beta_{0}$ are the polar angle and azimuthal angle with respect to the laser polarized direction; $F$ is the Fourier transform of the scattering amplitude $f$, and is proportional to the atomic potential [39]. $F_{j}(-\vec{r}) \otimes F_{k}(\vec{r})=F_{j}(\vec{r}) \star F_{k}(\vec{r})$, where $\otimes$ signifies convolution and $\star$ stands for correlation. Using the definition of a delta function in spherical coordinates $\quad \delta\left[\vec{r}-\vec{r}_{j k}\left(\alpha_{0}, \beta_{0}\right)\right]=\frac{1}{r^{2} \sin \alpha} \delta\left(r-r_{j k}\right) \delta\left(\alpha-\alpha_{0}\right)$ $\delta\left(\beta-\beta_{0}\right)$,

$$
\begin{gathered}
\iint g_{j k}\left(\alpha_{0}\right) \sin \alpha_{0} \delta\left[\vec{r}-\vec{r}_{j k}\left(\alpha_{0}, \beta_{0}\right)\right] d \alpha_{0} d \beta_{0} \\
=\frac{g_{j k}(\alpha) \delta\left(r-r_{j k}\right)}{r_{j k}{ }^{2}} .
\end{gathered}
$$

Equation (C5a) becomes

$F T_{3 D}^{-1}\left[I_{\mathrm{mol}}(\vec{s})\right]=\sum_{j=1}^{n} \sum_{k=1, j \neq k}^{n} g_{j k}(\alpha) \delta\left(r-r_{j k}\right) \otimes \frac{F_{j}(\vec{r}) \star F_{k}(\vec{r})}{r_{j k}^{2}}$.

The $\delta\left(r-r_{j k}\right)$ are part of the pair distribution function (PDF), which has a peak with position corresponding to the distance between each pair of atoms in the molecule. $g(\alpha)$ is the angular distribution of the PDF in real-space. Equation ( $\mathrm{C} 5 \mathrm{c}$ ) shows that each component of the PDF is convolved with a correlation of the Fourier transform of the atomic form factors. Here we define the right-hand side of $(\mathrm{C} 5 \mathrm{c})$ as the modified pair distribution function (MPDF).

We now show how the molecular diffraction pattern measured on a 2D detector with limited dimensions relates to Eq. (C5c). Define a Cartesian coordinate system for the molecules in real-space, where the plane $x-y$ is parallel to the 2D detector $\left(s_{x}-s_{y}\right)$, the $y$ axis corresponds to the direction of the laser polarization, and the $z$ axis represents the direction of propagation of the incident electron beam. We define a truncating function $h(s)$ that corresponds to the effective measurement area (detector area in this case), which has the common form $h(s)=1$ for $s \leqslant s_{\max }$, and $h(s)=0$ for $s>s_{\max }$, with its Fourier inversion $H(r)$. For small-angle diffraction experiments, the momentum transfer component along the $\mathrm{z}$ axis can be approximated to be zero, $s_{z} \cong 0$. Define $I_{\mathrm{mol}}^{E}(\vec{s})=h(s) \cdot I_{\mathrm{mol}}(\vec{s})$, and $I_{\mathrm{mol}}^{E}(\vec{s})$ measured on a flat detector is $I_{\mathrm{mol}}^{E}\left(s_{x}, s_{y}, s_{z}=0\right)=h\left(s_{x}, s_{y}, s_{z}=0\right)$. $I_{\mathrm{mol}}\left(s_{x}, s_{y}, s_{z}=0\right)$. Replacing $I_{\mathrm{mol}}(\vec{s})$ with $I_{\mathrm{mol}}^{E}(\vec{s})$ in $(\mathrm{C} 5 \mathrm{c})$, and using (C3b), we have

$$
\begin{aligned}
& \operatorname{Abel}^{-1} \mathrm{FT}_{2 D}^{-1}\left[I_{\mathrm{mol}}^{E}\left(s_{x}, s_{y}, s_{z} \cong 0\right)\right] \\
& =H(r) \otimes \sum_{j=1}^{n} \sum_{k=1, j \neq k}^{n} g_{j k}(\alpha) \delta\left(r-r_{j k}\right) \otimes \frac{F_{j}(\vec{r}) \star F_{k}(\vec{r})}{r_{j k}^{2}}, \\
& \mathrm{Abel}^{-1} \mathrm{FT}_{2 D}^{-1}\left[I_{\mathrm{mol}}^{E}\left(s_{x}, s_{y}, s_{z} \cong 0\right)\right] \\
& \quad=\sum_{j=1}^{n} \sum_{k=1, j \neq k}^{n} g_{j k}(\alpha) H\left(r-r_{j k}\right) \otimes \frac{F_{j}(\vec{r}) \star F_{k}(\vec{r})}{r_{j k}{ }^{2}} .
\end{aligned}
$$

The $H\left(r-r_{j k}\right)$ are terms of the measured pair distribution function (PDF), indicating the spatial resolution of the setup. Using the symmetry of the alignment with respect to the laser polarization, the three-dimensional MPDF is shown in two-dimensional form by the use of $\vec{r}=(\rho, y)$, where $\rho=$ $\sqrt{x^{2}+z^{2}}$ and $\tan \alpha=\frac{\rho}{y}$.

\section{APPENDIX D: DATA ANALYSIS}

Here we describe the steps for processing the experimental data. First, hot pixels are removed from each image, and each experimental image is normalized by the total counts. The anisotropy is calculated by dividing the image into horizontal and vertical cones with respect to the direction of the laser polarization (vertical), with opening angle of $60^{\circ}$, and $s$ ranging from 3 to $4.5 \AA^{-1}$. The difference-diffraction patterns are calculated with $\Delta I_{\mathrm{mol}}(\mathrm{s}, t)=I(\mathrm{~s}, t)-I(s, t<-1 \mathrm{ps})$ for each image, and are symmetrized over four quadrants. A beam stop, held by a thin wire, is used to block the transmitted electron beam. The minimum value of momentum transfer, $s$, captured in the experiment is $1.2 \AA^{-1}$. The maximum value is $6.0 \AA^{-1}$, limited by the size of the detector. The data blocked by the beam stop is extrapolated smoothly to zero at the origin. The data behind the wire are replaced using the symmetry of the image before extracting the angular distribution. The molecular diffraction intensity, $I_{\mathrm{mol}}(\boldsymbol{s}, t)$, is calculated by adding the simulated random molecular scattering to $\Delta I_{\text {mol }}(s, t)$, after rescaling by a factor of 0.35 . The MPDF is constructed following the steps in Appendix C. Zero padding is used to improve the sampling of the Fourier transforms in the region of interest, and a Gaussian damping function with rms 
width of $6 \AA^{-1}$ is applied to suppress noise at high $s$ as well as minimize the effects of the discontinuity caused by the limited detector range. The inversion result is converted to polar coordinates to extract the angular distribution $g(\alpha)$. A baseline is added to remove the positive offset caused by the convolutions in MPDF. The angular distribution is normal- ized by the equation $\int_{0}^{\pi} g(\alpha) \sin \alpha d \alpha=1$. The theoretical angular distribution is calculated using a simulation of the alignment process, as described in Appendix C. The simulated diffraction-difference patterns, molecular diffraction, and inversions are generated with the same procedures as the experimental data.
[1] A. H. Zewail, Angew. Chem., Int. Ed. 39, 2586 (2000).

[2] A. Stolow, A. E. Bragg, and D. M. Neumark, Chem. Rev. 104, 1719 (2004).

[3] H. Ihee, V. A. Lobastov, U. M. Gomez, B. M. Goodson, R. Srinivasan, C.-Y. Ruan, and A. H. Zewail, Science 291, 458 (2001).

[4] C. J. Hensley, J. Yang, and M. Centurion, Phys. Rev. Lett. 109, 133202 (2012).

[5] M. P. Minitti, J. M. Budarz, A. Kirrander, J. S. Robinson, D. Ratner, T. J. Lane, D. Zhu, J. M. Glownia, M. Kozina, H. T. Lemke, M. Sikorski, Y. Feng, S. Nelson, K. Saita, B. Stankus, T. Northey, J. B. Hastings, and P. M. Weber, Phys. Rev. Lett. 114, 255501 (2015).

[6] S. Chelkowski, P. B. Corkum, and A. D. Bandrauk, Phys. Rev. Lett. 82, 3416 (1999).

[7] M. Burt, R. Boll, Jason W. L. Lee, K. Amini, H. Köckert, C. Vallance, A. S. Gentleman, S. R. Mackenzie, S. Bari, C. Bomme et al., Phys. Rev. A 96, 043415 (2017).

[8] C. I. Blaga, J. Xu, A. D. DiChiara, E. Sistrunk, K. Zhang, P. Agostini, T. A. Miller, L. F. DiMauro, and C. D. Lin, Nature 483, 194 (2012).

[9] B. Wolter et al., Science 354, 308 (2016).

[10] S. Germán and R. J. D. Miller, Rep. Prog. Phys. 74, 096101 (2011).

[11] A. H. Zewail, Annu. Rev. Phys. Chem. 57, 65 (2006).

[12] J. Yang, J. Beck, C. J. Uiterwaal, and M. Centurion, Nat. Commun. 6, 8172 (2015).

[13] F. Qi. Z. Ma, L. Zhao, Y. Cheng, W. Jiang, C. Lu, T. Jiang, D. Qian, Z. Wang, W. Zhang, P. Zhu, X. Zou, W. Wan, D. Xiang, and J. Zhang, Phys. Rev. Lett. 124, 134803 (2020).

[14] S. P. Weathersby et al., Rev. Sci. Instrum. 86, 073702 (2015).

[15] X. Yang, J. Li, M. Fedurin, V. Smaluk, L. Yu, L. Wu, W. Wan, Y. Zhu, and T. Shaftan, Sci. Rep. 9, 17223 (2019).

[16] H. W. Kim et al., Nat. Photonics 14, 245 (2020).

[17] J. Yang et al., Nat. Commun. 7, 11232 (2016).

[18] J. Yang et al., Science 361, 64 (2018).

[19] J. Yang, M. Guehr, X. Shen, R. Li, T. Vecchione, R. Coffee, J. Corbett, A. Fry, N. Hartmann, C. Hast, K. Hegazy, K. Jobe, I. Makasyuk, J. Robinson, M. S. Robinson, S. Vetter, S.
Weathersby, C. Yoneda, X. Wang, and M. Centurion, Phys. Rev. Lett. 117, 153002 (2016).

[20] K. J. Wilkin, R. M. Parrish, J. Yang, T. J. A. Wolf, J. P. F. Nunes, M. Guehr, R. Li, X. Shen, Q. Zheng, X. Wang, T. J. Martinez, and M. Centurion, Phys. Rev. A 100, 023402 (2019).

[21] T. J. A. Wolf et al., Nat. Chem. 11, 504 (2019).

[22] J. Yang et al., Science 368, 885 (2020).

[23] T. van Oudheusden, P. L. E. M. Pasmans, S. B. van der Geer, M. J. de Loos, M. J. van der Wiel, and O. J. Luiten, Phys. Rev. Lett. 105, 264801 (2010).

[24] O. Zandi, K. J. Wilkin, Y. Xiong, and M. Centurion, Struct. Dyn. 4, 044022 (2017).

[25] M. R. Otto, L. P. René de Cotret, M. J. Stern, and B. J. Siwick, Struct. Dyn. 4, 051101 (2017).

[26] P. Baum and A. H. Zewail, Proc. Natl. Acad. Sci. USA 103, 16105 (2006).

[27] P. Zhang, J. Yang, and M. Centurion, New J. Phys. 16, 083008 (2014).

[28] X. Ren, V. Makhija, and V. Kumarappan, Phys Rev Lett 112, 173602 (2014).

[29] H. Stapelfeldt, Eur. Phys. J. D 26, 15 (2003).

[30] L. Holmegaard, J. H. Nielsen, I. Nevo, H. Stapelfeldt, F. Filsinger, J. Kupper, and G. Meijer, Phys. Rev. Lett. 102, 023001 (2009).

[31] I. Nevo, L. Holmegaard, J. H. Nielsen, J. L. Hansen, H. Stapelfeldt, F. Filsinger, G. Meijer, and J. Kupper, Phys. Chem. Chem. Phys. 11, 9912 (2009).

[32] E. T. Karamatskos et al., Nat. Commun. 10, 3364 (2019).

[33] T. Seideman and E. Hamilton, Adv. At., Mol. Opt. Phys. 52, 289 (2006).

[34] J. Ortigoso, M. Rodríguez, M. Gupta, and B. Friedrich, J Chem. Phys. 110, 3870 (1999).

[35] O. F. Hagena, Surf. Sci. 106, 101 (1981).

[36] X. Shen et al., Struct. Dyn. 6, 054305 (2019).

[37] J. Yang et al., Faraday Discuss. 194, 563 (2016).

[38] See Supplemental Material at http://link.aps.org/supplemental/ 10.1103/PhysRevResearch.2.043064 for the experimental and calculated movies of the rotational wavepacket dynamics.

[39] L. O. Brockway, Rev. Mod. Phys. 8, 231 (1936). 